\title{
Skeletal Muscle MRI in Complex Regional Pain Syndrome
}

\author{
Yoichiro Nishida ${ }^{1}$, Yuki Saito ${ }^{1}$, Takanori Yokota ${ }^{1}$, Takashi Kanda ${ }^{1,2}$ and Hidehiro Mizusawa ${ }^{1}$
}

\begin{abstract}
Background Conventional magnetic resonance imaging (MRI) sequences of patients with complex regional pain syndrome (CRPS) have shown abnormal signals in skin, soft tissue, joints, bone, and bone marrow, but not yet in skeletal muscles, during the acute phase. The aim of this study was to clarify whether or not the affected muscles in CRPS patients show abnormal MRI signal intensities or signal enhancement by gadolinium dimeglumine during the acute phase.

Patients and Methods MRI studies of skeletal muscles were performed on three patients of CRPS. Out of three patients, MRI was performed on three at stage 1, one in improving phase, two in remission phase, and one at stage 3. MRI was performed in the transaxial plane with both T2-weighted imaging (T2WI) and fatsuppressed T1-weighted imaging (T1WI) with or without gadolinium dimeglumine enhancement.

Results All patients at stage 1 showed hyperintense muscle signals on T2WI and gadolinium dimeglumine enhancement on T1WI. Following clinical improvement, the hyperintense lesions reverted to near normal. Muscles in the chronic phase showed high signals on both T2WI and T1WI without gadolinium dimeglumine enhancement.

Conclusion MRI abnormalities in the acute phase are consistent with muscular edema, interstitial edema, and vascular hyperpermeability. These MRI findings suggest the presence of hemodynamic abnormalities caused by microangiopathy, sympathetic abnormalities, or both, which may lead to ischemia of affected muscles. Chronic phase abnormalities indicated the presence of muscle atrophy and fibrosis or fatty infiltration of the affected muscle.
\end{abstract}

Key words: acute phase, complex regional pain syndrome, magnetic resonance imaging, microangiopathy, muscle, reflex sympathetic dystrophy

(Inter Med 48: 209-212, 2009)

(DOI: 10.2169/internalmedicine.48.1611)

\section{Introduction}

Complex regional pain syndrome (CRPS), which has also been called as reflex sympathetic dystrophy (RSD), causalgia, Sudeck's atrophy, algodystrophy and shoulder-hand syndrome, presents as an array of painful conditions that are characterized by a continuing regional pain that is seemingly disproportionate in time or degree to the usual course of any known trauma or other lesion (1). The pain is regional and usually has a distal predominance of abnormal sensory, motor, sudomotor, vasomotor, and/or trophic findings. CRPS is commonly divided into three stages. Stage 1 , the inflammatory stage, lasts a few months and is characterized by dif- fuse locoregional burning pain, edema, redness, and warmth. In stage 2, the dystrophic stage, pain during exercise, decreased skin temperature, hair loss, and the beginning of skin and muscle atrophy are shown. Stage 3, the atrophic stage, is characterized by persistent pain on exercise, scleroderma-like skin changes, and muscle atrophy. Some patients with stage 1 or 2 CRPS show marked improvements after treatment with sympathetic ganglion block, nonsteroidal anti-inflammatory drugs, or corticosteroids. Excluding conversion disorder can be difficult in the acute phase, when the differential diagnosis must be made with non-invasive techniques, such as magnetic resonance imaging (MRI). MRI is one of the noninvasive techniques and its application has greatly increased the sensitivity and accuracy of radio-

${ }^{1}$ Department of Neurology and Neurological Science, Graduate School, Tokyo Medical and Dental University, Tokyo and ${ }^{2}$ Department of Neurology and Clinical Neuroscience, Yamaguchi University Graduate School of Medicine, Ube Received for publication August 20, 2008; Accepted for publication September 30, 2008 Correspondence to Dr. Takashi Kanda, tkanda@yamaguchi-u.ac.jp 
logical diagnosis of various disorders. Nuclear magnetic resonance (NMR) spectroscopy reveals changes in skeletal muscle during the acute phase of CRPS (2), and conventional MRI indicates mainly muscle atrophy, fibrosis, or fatty infiltration in the chronic phase (3-5). We report the features of skeletal muscles in CRPS patients by conventional MRI, because it might prove useful for clinical investigation as well as give new insight into the pathophysiology of the disease.

\section{Patients and Methods}

\section{Patients}

CRPS diagnosis and staging were based on characteristic clinical symptoms and examination. Patients showed increased uptake of technetium methylene diphosphonate $\left({ }^{99 m}\right.$ Tc-MDPT) on bone scintigraphy, and changes in skin temperature, blood flow, or sudomotor function. Patients with other neurological disorders, relevant comorbidity and conversion disorder, were excluded. Three patients participated in this study, and their age, sex, disease trigger, affected limb, clinical staging, and disease duration at the time of MRI are shown in Table 1. MRI was performed on three at stage 1 , one in the improving phase, two in the remission phase, and one at stage 3 .

\section{Patient 1}

A 31-year-old man drove his truck on a highway and rear-ended a car. He was so panicky that he did not realize that a part of his body was injured. Three days after the accident, he became unable to walk because of severe pain in his left leg. His left foot became reddish and edematous. He was diagnosed as CRPS but his clinical symptoms barely improved despite many therapeutic trials. Crural muscular MRI was taken at stages 1 and 3 (two and 22 months from symptom onset, respectively).

\section{Patient 2}

A few days after blood was drawn from the cutaneous vein on her right forearm, a 24-year-old woman felt burning pain and allodynia with a central focus on the elbow. All her right fingers became edematous. Active and passive movements of right upper limb were severely restricted by the severe pain. The diagnosis of CRPS was made and therapeutic trials including blockade of stellate ganglion, oral steroid and nonsteroidal anti-inflammatory drugs (NSAIDs) improved her clinical symptoms. Forearm muscular MRI was taken at stage 1 , in the improving phase and in the remission phase (three, five and 20 months from symptom onset, respectively).

\section{Patient 3}

A 65-year-old woman suffered swelling and pain in the right foot without any possible initiating triggers. Her skin became purplish red and she was diagnosed as CRPS. Her clinical symptoms were remitted by oral NSAIDs. Crural muscular MRI was done at stage 1 and in the remission phase (three and 13 months from symptom onset, respectively).

\section{MRI}

MRI studies of skeletal muscles using T1-weighted imaging (T1WI) and T2-weighted imaging with fast spin-echo technique (T2WI) in the transaxial plane were performed using a high field strength $(1.5 \mathrm{~T})$, superconducting General Electric Signa MRI system. In addition, fat-suppressed T1WI was performed before and after the administration of gadolinium dimeglumine. We visually determined the enhancement effect of the gadolinium dimeglumine by comparison with uninvolved limbs, and categorized signal change severities on T2WI in the affected muscle into four groups: normal, no high-intensity area; mild, $0 \%$ to $33 \%$ of the whole muscle area showed high intensity; moderate, $33 \%$ to $66 \%$; and severe, more than $66 \%$, which is a modified method of categorization from a previous one (6). Two authors (Y.N. and Y.S.) independently quantified these changes and yielded the same results.

\section{Results}

In patient 1 , diffuse, abnormally high T2WI signals were observed in some muscles of the affected lower limb at stage 1 (Fig. 1A and Table 1). These muscles also showed high signals on fat-suppressed T1WI only after the administration of gadolinium dimeglumine (Figs. 1B, 1C). Followup T2WI and T1WI at stage 3 showed reduced muscle volume in the affected leg and abnormal, linear areas of high signal intensity (Figs. 1D, 1E). The area of T2WI signal changes was reduced in some muscles (Table 1), and there was no enhancement by gadolinium dimeglumine (Fig. 1F). In patient 2 , diffuse, abnormally high signals were observed at stage 1 in some muscles of the right forearm, both on T2WI and on fat-suppressed T1WI after the administration of gadolinium dimeglumine. There were no abnormal muscular signals on non-enhanced T1WI. At 5 and 20 months after onset, the high-signal areas on T2WI were gradually reduced in size in parallel with clinical improvement, and gadolinium dimeglumine gave no signal enhancement (Table 1). Although the time course and appearance of the abnormal MRIs were similar in patients 1 and 2, the patients had different clinical outcomes. In patient 3 , some muscles of the affected leg showed diffuse high signals on T2WI at stage 1. Gadolinium dimeglumine enhanced fat-suppressed T1WI, although some muscles showed slightly higher signals on T1WI even without enhancement. The high-signal areas on T2WI were decreased, and the enhancement effect of gadolinium dimeglumine disappeared by 13 months after onset, which coincided with recovery (Table 1). 

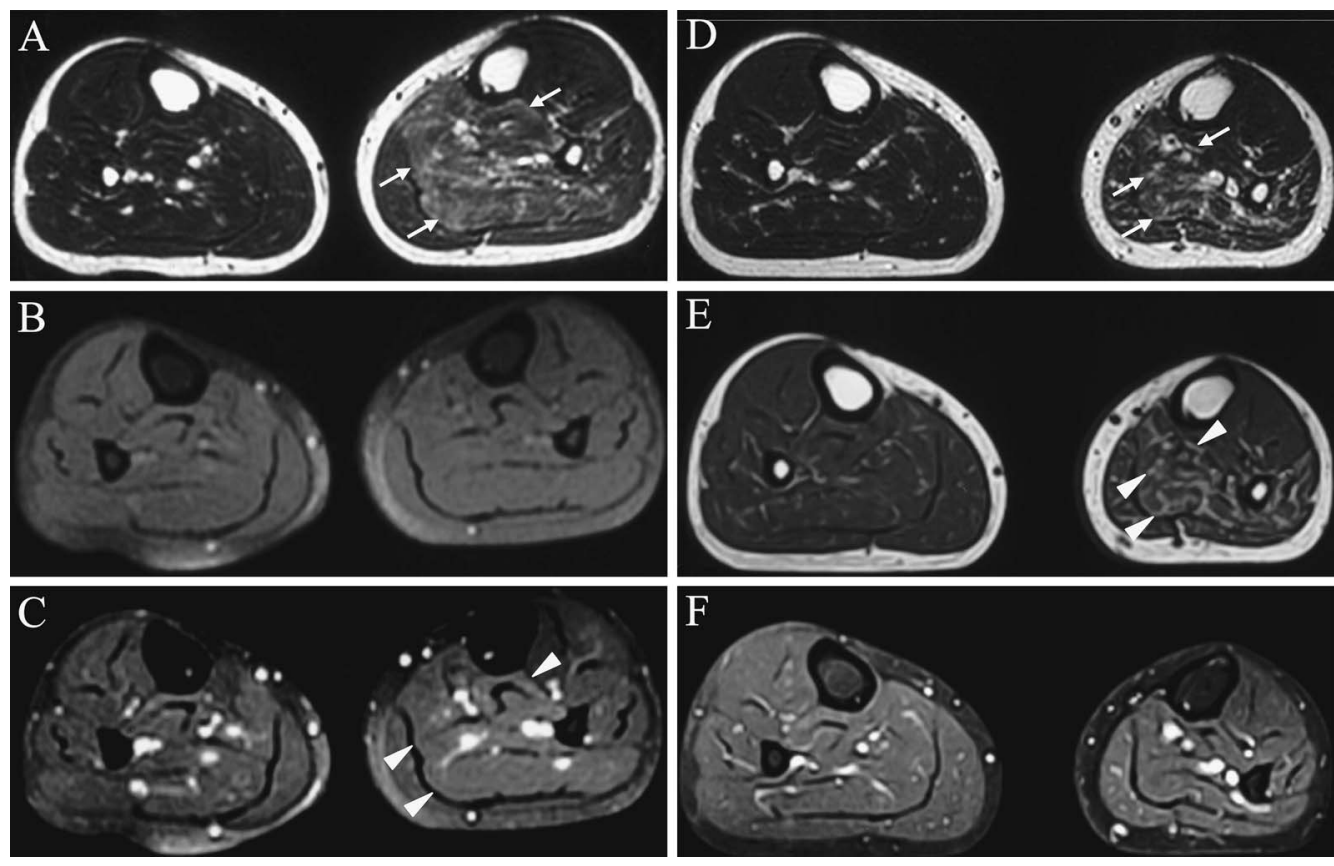

Figure 1. Lower limb muscle MRI of patient 1 in the acute phase at two months after onset (AC), and in the chronic phase at 22 months after onset (D-F). At stage 1, T2WI revealed diffuse, abnormally high signals in affected muscles in the left leg (arrows in A), while fat-suppressed T1WI was normal (B). Fat-suppressed T1WI after the administration of gadolinium dimeglumine showed enhancement on most of the muscles showing increased signals on T2WI (arrowheads in C). On the other hand, at stage 3, T2WI (arrows in D) and T1WI (arrowheads in E) revealed linear, abnormally high signals, and reduced muscle volume in the affected leg. Fat-suppressed T1WI revealed no enhancement by gadolinium dimeglumine $(F)$.

Table 1. Summary of Profiles and MRI Findings in Five Patients with CRPS

\begin{tabular}{|c|c|c|c|c|c|c|c|}
\hline \multirow[b]{3}{*}{$\begin{array}{l}\text { Patient } \\
\text { number }\end{array}$} & \multirow{2}{*}{\multicolumn{5}{|c|}{ Profiles }} & \multicolumn{2}{|l|}{ MRI } \\
\hline & & & & & & Distribution of affected muscles & \\
\hline & $\begin{array}{l}\text { Age } \\
\text { (Sex) }\end{array}$ & $\begin{array}{l}\text { Disease } \\
\text { trigger }\end{array}$ & $\begin{array}{c}\text { Affected } \\
\operatorname{limb(s)}\end{array}$ & $\begin{array}{l}\text { Clinical } \\
\text { stage }\end{array}$ & $\begin{array}{l}\text { Disease } \\
\text { duration }\end{array}$ & $\begin{array}{l}\text { and } \\
\text { High signals on T2WI in each muscle }\end{array}$ & $\begin{array}{c}\text { Enhancement } \\
\text { effect }\end{array}$ \\
\hline 1 & $\begin{array}{l}31 \\
(\mathrm{M})\end{array}$ & $\begin{array}{l}\text { traffic } \\
\text { accident }\end{array}$ & $\begin{array}{l}\text { left } \\
\mathrm{L} / \mathrm{E}\end{array}$ & $\begin{array}{l}\text { stage I } \\
\text { stage III }\end{array}$ & $\begin{array}{l}2 \text { months } \\
22 \text { months }\end{array}$ & $\begin{array}{l}\text { FDL, FHL, So, Pe , TP } \\
3+, 3+, 3+, 2+, 2+ \\
1+, 2+, 2+, 2+, 2+\end{array}$ & $\begin{array}{l}(+) \\
(-)\end{array}$ \\
\hline 2 & $\begin{array}{l}24 \\
(\mathrm{~F})\end{array}$ & $\begin{array}{l}\text { blood } \\
\text { drawing }\end{array}$ & $\begin{array}{l}\text { right } \\
\mathrm{U} / \mathrm{E}\end{array}$ & $\begin{array}{l}\quad \text { stage I } \\
\text { improving phase } \\
\text { remission phase }\end{array}$ & $\begin{array}{l}3 \text { months } \\
5 \text { months } \\
20 \text { months }\end{array}$ & $\begin{array}{rr}\text { APL/EPB, FDP, PT, } \mathrm{Su} \\
3+\quad, 3+, 3+, 2+ \\
3+ & , 3+, 2+, 1+ \\
1+ & , 2+, 2+, 1+\end{array}$ & $\begin{array}{l}(+) \\
(-) \\
(-)\end{array}$ \\
\hline 3 & $\begin{array}{l}65 \\
(\mathrm{~F})\end{array}$ & unclear & $\begin{array}{l}\text { right } \\
\mathrm{L} / \mathrm{E}\end{array}$ & $\begin{array}{l}\text { stage I } \\
\text { remission phase }\end{array}$ & $\begin{array}{l}3 \text { months } \\
13 \text { months }\end{array}$ & $\begin{array}{c}\text { FDL, FHL, TP, So } \\
3+, 3+, 3+, 2+ \\
1+, 1+, 2+, 1+\end{array}$ & $\begin{array}{c}(+) \\
(-)\end{array}$ \\
\hline
\end{tabular}

$\mathrm{U} / \mathrm{E}=$ upper extremity; L/E = lower extremity; $1+=$ mild signal changes; $2+=$ moderate signal changes; $3+=$ severe signal changes, as defined in the text; $(+)=$ present; $(-)=$ absent; FDL = flexor digitorum longus muscle; FHL = flexor hallucis longus muscle; $\mathrm{So}=$ soleus muscle; $\mathrm{Pe}=$ peroneus muscle; $\mathrm{TP}=$ tibialis posterior muscle; $\mathrm{APL} / \mathrm{EPB}=$ abductor pollicis longus and extensor pollicis brevis muscles; $\mathrm{FDP}=$ flexor digitorum profundus muscle; $\mathrm{PT}=$ pronator teres muscle; $\mathrm{Su}=$ supinator muscle.

\section{Discussion}

CRPS patients show abnormal MRI signals, either in T2WI, T1WI, or short-tau inversion recovery images, in skin, soft tissue, joints, bone, and bone marrow at various stages $(3-5,7)$. Only four reports refer to abnormal muscle MRI findings. Three of them primarily describe muscle atrophy, fibrosis, and fatty infiltration in later disease stages by conventional MRI sequences (3-5), and the other one shows abnormal muscular metabolism of high-energy phosphate by ${ }^{31} \mathrm{P}$ NMR spectroscopy (2). To date, no reports have dis- 
cussed the values of muscular abnormal signal changes in the acute phase shown by conventional MRI sequences. Here, we describe abnormal muscular signals in the acute phase of CRPS on T2WI and on T1WI with gadolinium enhancement. All three patients assessed at stage 1 showed hyperintense signals in affected muscles on T2WI, with most of these areas also showing enhancement with gadolinium dimeglumine on T1WI. These MRI features indicate that both edema and pathological capillary hyperpermeability occur in some muscles of the affected limb in the acute phase. In two patients in remission phase, these abnormal hyperintensities were reduced in size and less bright in parallel with the clinical improvement. MRI abnormalities did not correlate with outcomes, as patients 1 and 2 showed the same pattern of abnormal signals at stage 1 , although the clinical course of patient 1 worsened while that of patient 2 improved. In contrast, patients in the chronic phase showed only muscular fibrosis or fatty infiltration, which produced hyperintense signals on T2WI and T1WI without gadolinium enhancement.

The abnormal signal pattern of muscle MRI in the acute phase of inflammatory myopathies including dermatomyositis, polymyositis and inclusion body myositis resembles that in the acute phase of CRPS. The abnormal signal pattern of inflammatory myopathies is considered to reflect muscular edema $(8,9)$. Although the exact mechanism of these abnormal muscle signals in acute CRPS is not clear, they might result from microangiopathy; light and electron microscopic analysis of skeletal muscles showed impressive capillary changes, ranging from severely thickened basal membranes to necrosis in the chronic phase, suggesting the presence of microangiopathy in muscles (10). Hemodynamic abnormalities resulting from abnormal sympathetic function, and the resulting ischemia, could also cause these abnormal signals. These hemodynamic abnormalities could be caused by strong vasoconstriction of the sympathetically reinnervated blood vessels due to locally released vasoactive substances, or by systemically circulating catecholamines (11). Moreover, the abnormal signal patterns of muscle MRI in the chronic phases of CRPS and myopathies are also similar. In the chronic phase of myopathies, continuous inflammatory insult against skeletal muscles results in irreversible muscle changes, such as atrophy, which presents as decreased muscle volume on MRI, and fibrosis or fatty infiltration, which shows increased signals on T2WI and T1WI. Continuous insult against muscles would bring the same results in cases of CRPS, as well.

In addition, it is of interest that muscle MRI abnormalities in the acute phase of CRPS are not restricted to muscles innervated by specific peripheral nerves or spinal roots, but they have a similar distribution as in inflammatory myopathies. Abnormal signal muscles in CRPS are spread in some localized area, which is similar to inflammatory myopathies, while the affected muscles generally distribute bilateral, symmetric and proximal dominant in most of the inflammatory myopathies, but not in CRPS. These findings suggest that the pathological process in acute CRPS may progress in localized muscles and not through problems with circulation, innervation, or abnormal spinal reflexes.

In conclusion, we report that muscle MRI of CRPS patients in the acute phase showed hyperintense signals on T2WI and on T1WI with gadolinium enhancement. These MRI findings are consistent with the presence of hemodynamic abnormalities caused by microangiopathy, sympathetic abnormalities, or both, which may lead to ischemia of the affected muscles, spreading from localized to adjoining muscles in acute pathophysiology. Although further studies of larger numbers of patients are needed, muscle MRI could be useful for the diagnosis and staging of CRPS.

\section{References}

1. Harden RN, Bruehl S, Stanton-Hicks M, Wilson PR. Proposed new diagnostic criteria for complex regional pain syndrome. Pain Med 8: 326-331, 2007.

2. Heerschap A, den Hollander JA, Reynen H, Goris RJ. Metabolic changes in reflex sympathetic dystrophy: a ${ }^{31} \mathrm{P}$ NMR spectroscopy study. Muscle Nerve 16: 367-373, 1993.

3. Schweitzer ME, Mandel S, Schwartzman RJ, Knobler RL, Tahmoush AJ. Reflex sympathetic dystrophy revisited: MR imaging findings before and after infusion of contrast material. Radiology 195: 211-214, 1995.

4. Koch E, Hofer HO, Sialer G, Marincek B, von Schulthess GK. Failure of MR imaging to detect reflex sympathetic dystrophy of the extremities. Am J Roentgenol 156: 113-115, 1991.

5. Schellhas KP. MR imaging of muscles of mastication. Am J Roentgenol 153: 847-855, 1989.

6. Mercuri E, Lampe A, Allsop J, et al. Muscle MRI in Ullrich congenital muscular dystrophy and Bethlem myopathy. Neuromuscul Disord 15: 303-310, 2005.

7. Rankine JJ, Smith FW, Scotland TR. Case report: short tau inversion recovery (STIR) sequence MRI appearances of reflex sympathetic dystrophy. Clin Radiol 50: 188-190, 1995.

8. Petersilge CA. Imaging of muscle. In: Neuromuscular Disorders in Clinical Practice. Katirji B, Kaminski HJ, Preston DC, Eds. Butterworth Heinemann, Boston, 2002: 283-293.

9. Reimers CD, Vogl TJ. Inflammatory myopathies. In: Muscle Imaging in Health and Disease. Fleckenstein JL, Crues JV, Reimers CD, Eds. Springer, New York, 1996: 283-293.

10. van der Laan L, ter Laak HJ, Gabreels-Festen A, Gabreels F, Goris RJ. Complex regional pain syndrome type I (RSD): pathology of skeletal muscle and peripheral nerve. Neurology 51: 20-25, 1998.

11. Jänig W. Pain and the sympathetic nervous system: pathophysiological mechanisms. In: Autonomic Failure. A Textbook of Clinical Disorders of the Autonomic Nervous System. 4th ed. Mathias CJ, Bannister R, Eds. Oxford Univ. Press, New York, 2000: 99108.

\footnotetext{
(C) 2009 The Japanese Society of Internal Medicine http://www.naika.or.jp/imindex.html
} 